\title{
A NONUNIFORM BOUND ON CONVERGENCE TO NORMALITY
}

\author{
By C. C. HeYde \\ Australian National University
}

\begin{abstract}
Various asymptotically correct bounds on the uniform metric for distance between distribution functions in the central limit theorem for sums of independent and identically distributed random variables have previously been given. It is shown in the present paper that corresponding nonuniform bounds can be given for the difference between distribution functions. These results have much wider applicability, such as for obtaining probabilities of moderate deviation or for dealing with $L_{p}$ metrics, $1 \leqq p \leqq \infty$.
\end{abstract}

1. Introduction and results. Let $X_{i}, i=1,2, \ldots$ be independent and identically distributed random variables with $E X_{1}=0, E X_{1}^{2}=1$, and distribution function $F$. Write $S_{n}=\sum_{i=1}^{n} X_{i}, n \geqq 1, G(x)=P\left(\left|X_{1}\right| \leqq x\right), F_{n}(x)=P\left(S_{n} \leqq x n^{\frac{1}{2}}\right)$, and denote by $\Phi$ the distribution function of the unit normal law. We shall establish the following theorem.

Theorem 1. Suppose $E\left|X_{1}\right|^{2+\alpha}<\infty$ for some $0 \leqq \alpha \leqq 1$. Then for all $x$,

$$
\left|F_{n}(x)-\Phi(x)\right|<\left(1+|x|^{2+\alpha}\right)^{-1} c_{n}(\alpha),
$$

where

$$
c_{n}(\alpha)=C\left\{n^{-\frac{1}{2}} \int_{0}^{n^{\frac{1}{2}}} y^{3} d G(y)+n^{-\alpha / 2} \int_{n \frac{1}{2}}^{\infty} y^{2+\alpha} d G(y)\right\},
$$

$C$ being a universal constant. Furthermore, writing $\Delta_{n}=\sup _{x}\left|F_{n}(x)-\Phi(x)\right|$, the following results hold.

(i) The conditions $\sum n^{-1} \Delta_{n}<\infty$ and $\sum n^{-1} c_{n}(0)<\infty$ are equivalent and hold if and only if $E X_{1}^{2} \log \left(1+\left|X_{1}\right|\right)<\infty$.

(ii) For $0<\delta<1$, the conditions $\sum n^{-1+\delta / 2} \Delta_{n}<\infty$ and $\sum n^{-1+\delta / 2} c_{n}(0)<\infty$ are equivalent and hold if and only if $E\left|X_{1}\right|^{2+\delta}<\infty$.

(iii) For $0<\delta<1$, the conditions $\Delta_{n}=O\left(n^{-\delta / 2}\right)$ and $c_{n}(0)=O\left(n^{-\delta / 2}\right)$ as $n \rightarrow \infty$ are equivalent and hold if and only if $\int_{z}^{\infty} x^{2} d G(x)=O\left(z^{-\delta}\right)$ as $z \rightarrow \infty$.

This theorem extends results of Heyde [2] and of Ibragimov [5] which deal with the uniform metric in (i), (ii) and (iii). The result is useful for many purposes, such as for obtaining probabilities of moderate deviation (e.g. to generalize Theorem 4 of Davis [1]), for obtaining results on $L_{p}$ metrics, $1 \leqq p<\infty$, for departure from normality or for estimating differences of the kind $E b\left(S_{n}\right)-$ $E b\left(n^{\frac{1}{2}} Y\right)$ for large $n$ and suitable functions $b, Y$ having a unit normal distribution. A sample application, establishing a similarity of behaviour of the $L_{p}$ metrics,

Received November 4, 1974; revised March 16, 1975.

AMS 1970 subject classifications. Primary 60F05, 60G50; Secondary 60F 10.

Key words and phrases. Central limit theorem, convergence rates, independent random variables, nonuniform convergence, probabilities of moderate deviations, $L_{p}$ metrics. 
$1 \leqq p \leqq \infty$, is given in the following corollary which extends Theorem 2 of Heyde [3].

Corollary 2. Put

$$
\begin{aligned}
& \left\|F_{n}-\Phi\right\|_{p}=\left(\int_{-\infty}^{\infty}\left|F_{n}(x)-\Phi(x)\right|^{p} d x\right)^{1 / p}, \quad 1 \leqq p<\infty, \\
& \left\|F_{n}-\Phi\right\|_{\infty}=\sup _{x}\left|F_{n}(x)-\Phi(x)\right| .
\end{aligned}
$$

Then, for $0 \leqq \delta<1$ and $1 \leqq p \leqq \infty$, the following two conditions are equivalent:

(a) $E X_{1}^{2} \log \left(1+\left|X_{1}\right|\right)<\infty$ if $\delta=0, E\left|X_{1}\right|^{2+\delta}<\infty$ if $0<\delta<1$,

(b) $\sum n^{-1+\delta / 2}\left\|F_{n}-\Phi\right\|_{p}<\infty$.

It should be remarked that the complementary result to this corollary which deals with the case of $\left\|F_{n}-\Phi\right\|_{p}=O\left(n^{-\delta \cdot 2}\right), 0<\delta<1$, has been obtained, by quite different methods, in Theorem 4.3 of Ibragimov [5].

2. Proofs. To prove Theorem 1 we start by taking $x \geqq 2$ for definiteness. The case $x \leqq-2$ can then be dealt with by replacing the $X_{i}$ 's by $-X_{i}$ 's and the case $|x|<2$ will follow from a bound on the uniform metric of Osipov and Petrov [7].

Put

$$
\begin{aligned}
X_{k}^{(n)}(x) & =X_{k} & & \text { if } \quad\left|X_{k}\right| \leqq n^{\frac{1}{2}} x, \\
& =0 & & \text { otherwise, }
\end{aligned}
$$

and write $S_{n}^{(n)}(x)=\sum_{k=1}^{n} X_{k}^{(n)}(x)$. We have

$$
\begin{aligned}
F_{n}(x) & =P\left(S_{n} \leqq n^{\frac{1}{2}} x, S_{n}{ }^{(n)}(x) \leqq n^{\frac{1}{2}} x\right)+P\left(S_{n} \leqq n^{\frac{1}{2}} x, S_{n}{ }^{(n)}(x)>n^{\frac{1}{2}} x\right) \\
& \leqq P\left(S_{n}{ }^{(n)}(x) \leqq n^{\frac{1}{2}} x\right)+n P\left(\left|X_{1}\right|>n^{\frac{1}{2}} x\right)
\end{aligned}
$$

and similarly

$$
1-F_{n}(x) \leqq P\left(S_{n}^{(n)}(x)>n^{\frac{1}{2}} x\right)+n P\left(\left|X_{1}\right|>n^{\frac{1}{2}} x\right)
$$

so that

and hence

$$
F_{n}(x) \geqq P\left(S_{n}^{(n)}(x) \leqq n^{\frac{1}{2}} x\right)-n P\left(\left|X_{1}\right|>n^{\frac{1}{2}} x\right)
$$

$$
\left|F_{n}(x)-\Phi(x)\right| \leqq\left|P\left(S_{n}{ }^{(n)}(x) \leqq n^{\frac{1}{2}} x\right)-\Phi(x)\right|+n P\left(\left|X_{1}\right|>n^{\frac{1}{2}} x\right) .
$$

Now put $\mu_{n}(x)=E X_{1}^{(n)}(x), \sigma_{n}{ }^{2}(x)=\operatorname{Var} X_{1}^{(n)}(x)$. We have, writing

$$
\begin{aligned}
& y_{n}=\left[\sigma_{n}(x)\right]^{-1} n^{-\frac{1}{2}}\left[n^{\frac{1}{2}} x-n \mu_{n}(x)\right], \\
& \begin{aligned}
\mid P\left(S_{n}{ }^{(n)}(x)\right. & \left.\leqq n^{\frac{1}{2}} x\right)-\Phi(x) \mid \\
\leqq & P\left(\left[\sigma_{n}(x)\right]^{-1}\left(S_{n}{ }^{(n)}(x)-n \mu_{n}(x)\right) \leqq n^{\frac{1}{2}} y_{n}\right)-\Phi\left(y_{n}\right) \mid \\
& \quad+\left|\Phi\left(y_{n}\right)-\Phi\left(\left[\sigma_{n}(x)\right]^{-1} x\right)\right|+\left|\Phi\left(\left[\sigma_{n}(x)\right]^{-1} x\right)-\Phi(x)\right|
\end{aligned}
\end{aligned}
$$

But, using Theorem 2 of Nagaev [6],

$$
\begin{aligned}
\mid P\left([ \sigma _ { n } ( x ) ] ^ { - 1 } \left(S_{n}^{(n)}(x)\right.\right. & \left.\left.-n \mu_{n}(x)\right) \leqq n^{\frac{1}{2}} y_{n}\right)-\Phi\left(y_{n}\right) \mid \\
& \leqq C E\left|X_{1}^{(n)}(x)-\mu_{n}(x)\right|^{3} n^{-\frac{1}{2}}\left[\sigma_{n}(x)\right]^{-3}\left(1+\left|y_{n}\right|^{3}\right)^{-1} \\
& \leqq 8 C E\left|X_{1}^{(n)}(x)\right|^{3} n^{-\frac{1}{2}}\left|x-n^{\frac{1}{2}} \mu_{n}(x)\right|^{-3}
\end{aligned}
$$

$C$ denoting a universal constant. 
Now, for $x \geqq 2$ we have

$$
\left|\mu_{n}(x)\right|=\left|\int_{|y|>n \frac{1}{2} x} y d F(x)\right| \leqq \int_{n \frac{1}{2} x}^{\infty} y d G(x) \leqq n^{-\frac{1}{2}} x^{-1} \int_{n \frac{1}{2} x}^{\infty} y^{2} d G(y) \leqq n^{-\frac{1}{2}} x^{-1},
$$

so that

$$
n^{\frac{1}{2}}\left|\mu_{n}(x)\right| \leqq x^{-1} \leqq \frac{1}{2} x, \quad\left|x-n^{\frac{1}{2}} \mu_{n}(x)\right|^{3} \geqq \frac{1}{8} x^{3},
$$

and hence, from (5),

$$
\begin{aligned}
\mid P\left(\left[\sigma_{n}(x)\right]^{-1}\right. & \left.\left(S_{n}^{(n)}(x)-n \mu_{n}(x)\right) \leqq n^{\frac{1}{2}} y_{n}\right)-\Phi\left(y_{n}\right) \mid \\
& \leqq 64 C E\left|X_{1}^{(n)}(x)\right|^{3} n^{-\frac{1}{2}} x^{-3} \\
& =64 C n^{-\frac{1}{2}} x^{-3}\left\{\int_{0}^{n^{\frac{1}{2}}} y^{3} d G(y)+\int_{n^{\frac{1}{2}}}^{n^{\frac{1}{2}} x} y^{3} d G(y)\right\} \\
& \leqq 64 C n^{-\frac{1}{2}} x^{-3}\left\{\int_{0}^{n^{\frac{1}{2}}} y^{3} d G(y)+\left(n^{\frac{1}{2}} x\right)^{1-\alpha} \int_{n^{\frac{1}{2}}}^{\infty} y^{2+\alpha} d G(y)\right\} \\
& \leqq 64 C x^{-(2+\alpha)}\left\{n^{-\frac{1}{2}} \int_{0}^{n^{\frac{1}{2}}} y^{3} d G(y)+n^{-\alpha \cdot 2} \int_{n \frac{1}{2}}^{\infty} y^{2+\alpha} d G(y)\right\} .
\end{aligned}
$$

Further, for $x \geqq 1$,

$$
\begin{aligned}
n P\left(\left|X_{1}\right|>n^{\frac{1}{2}} x\right) & \leqq x^{-(2+\alpha)} n^{-\alpha / 2} \sup _{u \geqq n \frac{1}{2}} u^{2+\alpha} P\left(\left|X_{1}\right|>u\right) \\
& \leqq x^{-(2+\alpha)} n^{-\alpha / 2} \sup _{u \geqq n \frac{1}{2}} \int_{u}^{\infty} y^{2+\alpha} d G(y) \\
& \leqq x^{-(2+\alpha)} n^{-\alpha / 2} \int_{n^{\frac{1}{2}}}^{\infty} y^{2+\alpha} d G(y)
\end{aligned}
$$

It now remains to bound the terms $\left|\Phi\left(y_{n}\right)-\Phi\left(\left[\sigma_{n}(x)\right]^{-1} x\right)\right|$ and $\mid \Phi\left(\left[\sigma_{n}(x)\right]^{-1} x\right)-$ $\Phi(x) \mid$ from (4). Since $e^{\frac{1}{2} u^{2}}>\frac{1}{2} u^{2}$ for $u>0$ we have

$$
\begin{aligned}
\left|\Phi\left(y_{n}\right)-\Phi\left(\left[\sigma_{n}(x)\right]^{-1} x\right)\right| & \leqq(2 \pi)^{-\frac{1}{2}} \int_{\left.(x-n) \frac{1}{2}\left|\mu_{n}(x)\right|\right)\left[\sigma_{n}(x)\right]^{-1}}^{x\left[\sigma_{n}(x) e^{-\frac{1}{2}} u^{2} d u\right.} \\
& \leqq 2(2 \pi)^{-\frac{1}{2}} \int_{\left(x-n \frac{1}{2}\left|\mu_{n}(x)\right|\right)\left[\sigma_{n}(x)\right]^{-1}}^{x-2} u^{-2} d u \\
& \leqq 4(2 \pi)^{-\frac{1}{2}} n^{\frac{1}{2}} x^{-2}\left|\mu_{n}(x)\right|
\end{aligned}
$$

using (6). Also,

$$
n^{\frac{1}{2}}\left|\mu_{n}(x)\right| \leqq n^{\frac{1}{2}} \int_{n^{\frac{1}{2} x}}^{\infty} y d G(y) \leqq x^{-1} \int_{n^{\frac{1}{2} x} x}^{\infty} y^{2} d G(y) \leqq x^{-1} \int_{2 n \frac{1}{2}}^{\infty} y^{2} d G(y)
$$

and hence, from (9) and (10),

$$
\begin{aligned}
\left|\Phi\left(y_{n}\right)-\Phi\left(\left[\sigma_{n}(x)\right]^{-1} x\right)\right| & \leqq 4(2 \pi)^{-\frac{1}{2}} x^{-3} \int_{2 n^{\frac{1}{2}}}^{\infty} y^{2} d G(y) \\
& \leqq 4(2 \pi)^{-\frac{1}{2}} x^{-(2+\alpha)} \int_{2 n^{\frac{1}{2}}}^{\infty} y^{2} d G(y) \\
& \leqq 4(2 \pi)^{-\frac{1}{2}} x^{-(2+\alpha)} n^{-\alpha / 2} \int_{n^{\frac{1}{2}}}^{\infty} y^{2+\alpha} d G(y) .
\end{aligned}
$$

Next, since $e^{\frac{1}{2} u^{2}}>\frac{1}{8} u^{4} \geqq \frac{1}{4} u^{3}$ for $u \geqq 2$, we have for $x \geqq 2$,

$$
\begin{aligned}
\left|\Phi\left(\left[\sigma_{n}(x)\right]^{-1} x\right)-\Phi(x)\right| & \leqq 4(2 \pi)^{-\frac{1}{2}} \int_{x}^{x\left[\sigma_{n}(x)\right]^{-1}} u^{-3} d u \\
& =2(2 \pi)^{-\frac{1}{2}} x^{-2}\left[1-\sigma_{n}^{2}(x)\right] \\
& \leqq 4(2 \pi)^{-\frac{1}{2}} x^{-2} \int_{n \frac{1}{2} x}^{\infty} y^{2} d G(y) \\
& \leqq 4(2 \pi)^{-\frac{1}{2}} x^{-(2+\alpha)} n^{-\alpha / 2} \int_{n^{\frac{1}{2} x}}^{\infty} y^{2+\alpha} d G(y) \\
& \leqq 4(2 \pi)^{-\frac{1}{2}} x^{-(2+\alpha)} n^{-\alpha / 2} \int_{2 n \frac{1}{2}}^{\infty} y^{2+\alpha} d G(y) .
\end{aligned}
$$

Hence, using (3), (4), (7), (8), (11) and (12), we have for $|x| \geqq 2$ that there exists a universal constant $C$ such that, a fortiori,

$$
\left|F_{n}(x)-\Phi(x)\right| \leqq C\left(1+|x|^{2+\alpha}\right)^{-1}\left\{n^{-\frac{1}{2}} \int_{0}^{n^{\frac{1}{2}}} y^{3} d G(y)+n^{-\alpha / 2} \int_{n^{\frac{1}{2}}}^{\infty} y^{2+\alpha} d G(y)\right\}
$$


Furthermore, the inequality of Osipov and Petrov [7] can be specialized to give (see (2) of Heyde [4] wherein we take $\tau_{n}=C_{n}=n^{\frac{1}{2}} ; K_{0}$ is a universal constant)

$$
\begin{array}{rl}
\left|F_{n}(x)-\Phi(x)\right| \leqq n & P\left(\left|X_{1}\right|>n^{\frac{1}{2}}\right)+K_{0} n^{-\frac{1}{2}}\left[\sigma_{n}(1)\right]^{-3} \int_{0}^{n \frac{1}{2}} y^{3} d G(y) \\
& +n^{\frac{1}{2}}(2 \pi)^{-\frac{1}{2}}\left[\sigma_{n}(1)\right]^{-1}\left|\int_{|y| \leqq n} y d F(y)\right| \\
& +(2 \pi e)^{-\frac{1}{2}} \int_{n^{\frac{1}{2}}}^{\infty} y^{2} d G(y),
\end{array}
$$

and noting (8), (10) and $\sigma_{n}(1) \rightarrow 1$ as $n \rightarrow \infty$, we observe that the right hand side of (14) is upper bounded by the right hand side of (13) for suitable universal $C$ and $|x|<2$. This ensures that (1) also holds for $|x|<2$ and hence completes the proof of (1). In order to complete the remainder of the proof we first obtain a simple bound for $c_{n}(0)$.

Write $L(y)=\int_{y}^{\infty} x^{2} d G(x)$. Then

$$
\begin{aligned}
n^{-\frac{1}{2}} \int_{0}^{n^{\frac{1}{2}}} y^{3} d G(y) & =-n^{-\frac{1}{2}} \int_{0}^{n^{\frac{1}{2}}} y d L(y) \\
& \leqq n^{-\frac{1}{2}} \int_{0}^{n^{\frac{1}{2}}} L(y) d y,
\end{aligned}
$$

and, since $L(y) \downarrow$ as $y$ increases,

$$
c_{n}(0) \leqq 2 C n^{-\frac{1}{2}} \int_{0}^{n \frac{1}{2}} L(y) d y .
$$

Now suppose $0 \leqq \delta<1$ and $E X_{1}^{2} \log \left(1+\left|X_{1}\right|\right)<\infty$ if $\delta=0, E\left|X_{1}\right|^{2+\delta}<\infty$ if $0<\delta<1$. Here and below we use $C$ to denote a positive universal constant which may differ from one expression to the next. Using (15), we have

$$
\begin{aligned}
\sum_{n=1}^{\infty} n^{-1+\delta / 2} c_{n}(0) & \leqq C \sum_{n=1}^{\infty} n^{-(3-\delta) / 2} \sum_{k=1}^{n} \int_{(k-1) \frac{1}{2}}^{k^{\frac{1}{2}}} L(y) d y \\
& \leqq C \sum_{n=1}^{\infty} n^{-(3-\delta) / 2} \sum_{k=1}^{n} L\left((k-1)^{\frac{1}{2}}\right)\left(k^{\frac{1}{2}}-(k-1)^{\frac{1}{2}}\right) \\
& \leqq C \sum_{k=1}^{\infty} k^{-\frac{1}{2}} L\left((k-1)^{\frac{1}{2}}\right) \sum_{n=k}^{\infty} n^{-(3-\delta) / 2} \\
& \leqq C \sum_{k=1}^{\infty} k^{-1+\delta / 2} L\left((k-1)^{\frac{1}{2}}\right) \\
& =C \sum_{k=1}^{\infty} k^{-1+\delta / 2} \sum_{n=k}^{\infty} E\left\{X_{1}^{2} I\left((n-1)^{\frac{1}{2}}<\left|X_{1}\right| \leqq n^{\frac{1}{2}}\right)\right\} \\
& \leqq C \sum_{n=1}^{\infty} n^{\delta / 2} E\left\{X_{1}^{2} I\left((n-1)^{\frac{1}{2}}<\left|X_{1}\right| \leqq n^{\frac{1}{2}}\right)\right\} \\
& \leqq C E\left|X_{1}\right|^{2+\delta}<\infty, \quad \text { if } \quad 0<\delta<1, \quad \text { or }, \\
& \leqq C \sum_{n=1}^{\infty} \log (n+1) E\left\{X_{1}{ }^{2} I\left((n-1)^{\frac{1}{2}}<\left|X_{1}\right| \leqq n^{\frac{1}{2}}\right)\right\} \\
& \leqq C E X_{1}^{2} \log \left(1+\left|X_{1}\right|\right)<\infty, \quad \text { if } \delta=0 .
\end{aligned}
$$

Conversely, if $0 \leqq \delta<1$ and $\sum n^{-1+\delta / 2} c_{n}(0)<\infty$, then (2) gives $\sum n^{-1+\delta / 2} L\left(n^{\frac{1}{2}}\right)<\infty$ and

$$
\begin{aligned}
\sum_{n=1}^{\infty} n^{-1+\delta / 2} L\left(n^{\frac{1}{2}}\right) & =\sum_{n=1}^{\infty} n^{-1+\delta / 2} \sum_{k=n}^{\infty} E\left\{X_{1}^{2} I\left(k^{\frac{1}{2}}<\left|X_{1}\right| \leqq(k+1)^{\frac{1}{2}}\right)\right\} \\
& =\sum_{k=1}^{\infty} E\left\{X_{1}^{2} I\left(k^{\frac{1}{2}}<\left|X_{1}\right| \leqq(k+1)^{\frac{1}{2}}\right)\right\} \sum_{n=1}^{k} n^{-1+\delta / 2} \\
& \geqq C \sum_{k=1}^{\infty} k^{\delta / 2} E\left\{X_{1}^{2} I\left(k^{\frac{1}{2}}<\left|X_{1}\right| \leqq(k+1)^{\frac{1}{2}}\right)\right\} \\
& \geqq C E\left|X_{1}\right|^{2+\delta}, \quad \text { if } \quad 0<\delta<1, \quad \text { or } \\
& \geqq C \sum_{k=1}^{\infty} \log k E\left\{X_{1}^{2} I\left(k^{\frac{1}{2}}<\left|X_{1}\right| \leqq(k+1)^{\frac{1}{2}}\right)\right\} \\
& \geqq C E X_{1}^{2} \log \left(1+\left|X_{1}\right|\right), \quad \text { if } \delta=0 .
\end{aligned}
$$

The equivalence of $\sum n^{-1+\delta / 2} \Delta_{n}<\infty$ and $E X_{1}{ }^{2} \log \left(1+\left|X_{1}\right|\right)<\infty$ if $\delta=0$, 
$E\left|X_{1}\right|^{2+\delta}<\infty$ if $0<\delta<1$, follows from Heyde [2] and hence the proof of (i) and (ii) is complete.

Finally, to prove (iii) we first suppose that $L(y)=O\left(y^{-\delta}\right)$ as $y \rightarrow \infty$. Then, using $(15), c_{n}(0)=O\left(n^{-\delta / 2}\right)$. On the other hand, if $c_{n}(0)=O\left(n^{-\delta / 2}\right)$ then certainly, from (2),

$$
L\left(n^{\frac{1}{2}}\right)=\int_{n \frac{1}{2}}^{\infty} y^{2} d G(y)=O\left(n^{-\delta / 2}\right)
$$

which yields $L(y)=O\left(y^{-\delta}\right)$ as $y \rightarrow \infty$ as required. The equivalence of $\Delta_{n}=$ $O\left(n^{-\delta / 2}\right)$ and $L(z)=O\left(z^{-\delta}\right)$ as $z \rightarrow \infty$ was obtained by Ibragimov [5]. This completes the proof of Theorem 1 .

To establish Corollary 2 we first observe that the equivalence of (a) and (b) for $p=\infty$ follows from Theorem 1 as does the result that (a) implies (b) for $1 \leqq p<\infty$ since (a) gives $\sum n^{-1+\delta / 2} c_{n}(0)<\infty$ and $\left\|F_{n}-\Phi\right\|_{p}=O\left(c_{n}(0)\right)$. To see that (b) implies (a) note that the proof of the necessity part of the theorem of [2] is still applicable with minor modification (involving applications of Hölder's and Minkowski's inequalities if $p>1$ ).

Acknowledgment. I am indebted to J. R. Leslie for a helpful comment.

\section{REFERENCES}

[1] Davis, J. A. (1968). Convergence rates for probabilities of moderate deviations. Ann. Math. Statist. 39 2016-2028.

[2] HEYDE, C. C. (1967). On the influence of moments on the rate of convergence to the normal distribution. Z. Wahrscheinlichkeitstheorie und Verw. Gebiete 8 12-18.

[3] Heyde, C. C. (1969). Some properties of metrics in a study on convergence to normality. Z. Wahrscheinlichkietstheorie und Verw. Gebiete 11 181-192.

[4] Heyde, C. C. (1973). On the uniform metric in the context of convergence to normality. Z. Wahrscheinlichkeitstheorie und Verw. Gebiete 25 83-95.

[5] Ibragimov, I. A. (1966). On the accuracy of the Gaussian approximation to the distribution function of sums of independent random variables. Theor. Probability Appl. 11 559576.

[6] Nagaev, S. V. (1965). Some limit theorems for large deviations. Theor. Probability Appl. 10 214-235.

[7] Osipov, L. V. and Petrov, V. V. (1967). On an estimate of the remainder in the central limit theorem. Theor. Probability Appl. 12 281-286.

Department of Statistics

Australian National University P.O. BOX 4 CANBERRA

A.C.T. 2600

Australia 